\title{
Inflammation and glucose homeostasis are associated with specific structural features among adults without knee osteoarthritis: a cross-sectional study from the osteoarthritis initiative
}

\author{
Alina C. Stout ${ }^{1}$, Mary F. Barbe ${ }^{2}$, Charles B. Eaton ${ }^{3}$, Mamta Amin², Fatimah Al-Eid', Lori Lyn Price ${ }^{4,5}$, Bing Lu ${ }^{6}$,
} Grace H. Lo ${ }^{7,8}$, Ming Zhang ${ }^{1}$, Jincheng Pang ${ }^{9}$, Timothy E. McAlindon ${ }^{1}$ and Jeffrey B. Driban ${ }^{1 *}$

\begin{abstract}
Background: Greater age and body mass index are strong risk factors for osteoarthritis (OA). Older and overweight individuals may be more susceptible to OA because these factors alter tissue turnover in menisci, articular cartilage, and bone via altered glucose homeostasis and inflammation. Understanding the role of inflammation and glucose homeostasis on structural features of early-stage OA may help identify therapeutic targets to delay or prevent the onset of OA among subsets of adults with these features. We examined if serum concentrations of glucose homeostasis (glucose, glycated serum protein [GSP]) or inflammation (C-reactive protein [CRP]) were associated with prevalent knee bone marrow lesions (BMLs) or effusion among adults without knee OA.

Methods: We conducted a cross-sectional study using baseline data from the Osteoarthritis Initiative. We selected participants who had no radiographic knee OA but were at high risk for knee OA. Blinded staff conducted assays for CRP, GSP, and glucose. Readers segmented BML volume and effusion using semi-automated programs. Our outcomes were prevalent BML (knee with a BML volume $>1 \mathrm{~cm}^{3}$ ) and effusion (knee with an effusion volume $>7.5 \mathrm{~cm}^{3}$ ). We used logistic regression models with CRP, GSP, or glucose concentrations as the predictors. We adjusted for age, sex, body mass index (BMI), and Physical Activity Scale for the Elderly (PASE) scores.

Results: We included 343 participants: mean age $=59 \pm 9$ years, $\mathrm{BMI}=27.9 \pm 4.5 \mathrm{~kg} / \mathrm{m}^{2}$, PASE score $=171 \pm 82$, and $64 \%$ female. Only CRP was associated with BML prevalence (odds ratio $[\mathrm{OR}]=1.43,95 \%$ confidence interval $[\mathrm{Cl}]=1$. 09 to 1.87). For effusion, we found an interaction between BMI and CRP: only among adults with a BMI $<25 \mathrm{~kg} / \mathrm{m}^{2}$ was there a significant trend towards a positive association between CRP and effusion ( $O R=1.40,95 \% \mathrm{Cl}=1.00$ to 1.97). We detected a U-shaped relationship between GSP and effusion prevalence. Fasting glucose levels were not significantly associated with the presence of baseline effusion or BML.

Conclusions: Among individuals without knee OA, CRP may be related to the presence of BMLs and effusion among normal weight individuals. Abnormal GSP may be associated with effusion. Future studies should explore whether inflammation and glucose homeostasis are predictive of symptomatic knee OA.
\end{abstract}

Keywords: Bone marrow lesions, Effusion, Magnetic resonance imaging, Osteoarthritis

\footnotetext{
* Correspondence: jeffrey.driban@tufts.edu

'Division of Rheumatology, Tufts Medical Center, 800 Washington Street, Box

\#406, Boston, MA 02111, USA

Full list of author information is available at the end of the article
} 


\section{Background}

Greater age and body mass index are strong risk factors for osteoarthritis (OA) [1-4]. Older and overweight individuals may be more susceptible to OA because these factors alter tissue turnover in menisci, articular cartilage, and bone via altered glucose homeostasis [5-9] and inflammation [8, 10-14]. Individuals with osteoarthritis (OA) have greater systemic inflammation [15] and impaired glucose homeostasis [16]. However, it remains unclear if systemic inflammation and glucose homeostasis relate to specific structural features of early-stage OA, namely bone marrow lesions (BMLs) and effusion. BMLs and effusion are common features of OA that are associated with incidence of disease and symptoms [1720]. Specifically, BMLs may reflect changes in the subchondral bone (e.g., fibrosis, edema, necrosis, abnormal trabeculae) that may predispose the joint to articular cartilage loss and greater knee pain [17]. Meanwhile, effusion and synovitis are linked to incident radiographic knee OA up to 2 years prior to diagnosis [19]. BMLs and effusion are particularly interesting because they can change size in less than 12 weeks [21, 22]. Furthermore, clinical interventions may reduce BML size [23, 24] and effusion volume [22].

Understanding the role of inflammation and glucose homeostasis on structural features of early-stage OA may help identify therapeutic targets to delay or prevent the onset of OA among subsets of adults with these features. C-reactive protein (CRP) is a common clinical biomarker used to test systemic inflammation [25]. Individuals with greater CRP concentration are more likely to have greater OA-related symptoms [26] and develop incident radiographic $\mathrm{OA}$ [27]. In relation to specific structural features of knee OA, one study found that CRP may be associated with BMLs among adults with knee OA [28] but other investigators reported null findings among individuals with and without OA [29]. Hence, we evaluated CRP because it may play a role in OA pathophysiology, the biomarker is clinically available, and prior studies assessed CRP in relation to OA.

Despite evidence that metabolic syndrome and diabetes may be associated with OA there is limited evidence about the role of glucose homeostasis on BMLs or effusion [30]. To assess altered glucose homeostasis, we assessed glucose concentrations, which offers an assessment of glucose levels at the time of the study visit, and glycated serum protein (GSP), which provides a stable marker of glucose homeostasis over the 2 to 3 weeks prior to the study visit. It would be valuable to further explore the association between inflammation and glucose homeostasis among individuals without knee OA.

We sought to expand prior research by examining only individuals without radiographic knee OA. We assessed CRP and glucose concentrations, which had been tested in prior studies, and GSP, which provides a stable indicator of glucose homeostasis. The purpose of this study was to determine if serum concentrations of impaired glucose homeostasis (glucose or GSP) or systemic inflammation (CRP) were associated with the prevalence of knee BMLs or effusion. We focused on serum concentrations of these measures because serum would be easier to collect in most clinical settings than other samples (e.g., synovial fluid). Our long-term goal is to understand the relationship between glucose and/or inflammatory impairment and BMLs or effusion. This study represents a key early step to understanding whether systemic inflammation or irregular glucose homeostasis predisposes a knee to BMLs or effusion or whether BMLs and effusion lead to systemic inflammation and glucose irregularity.

\section{Methods}

The Osteoarthritis Initiative (OAI) is a longitudinal, multi-center, observational study of 4796 individuals with or at risk for knee OA at four sites in the United States: Memorial Hospital of Rhode Island, The Ohio State University, University of Maryland and Johns Hopkins University, and the University of Pittsburgh. The study staff enrolled men and women (45 to 79 years of age) between February 2004 and May 2006. Descriptions of the eligibility criteria and the OAI protocol are publicly available on the OAI website [31]. The OAI study was approved by institutional review boards at each OAI clinical site and the coordinating center: Memorial Hospital of Rhode Island Institutional Review Board, The Ohio State University's Biomedical Sciences Institutional Review Board, University of Pittsburgh Institutional Review Board, University of Maryland Baltimore - Institutional Review Board, and Committee on Human Research at University of California, San Francisco. All participants provided informed consent.

To determine if serum concentrations of impaired glucose homeostasis (glucose or GSP) or systemic inflammation (CRP) were associated with the prevalence of knee BMLs or effusion, we conducted a secondary analysis among a well characterized case-control sample that included three groups ( $n=125$ /group): development of accelerated knee OA (case), common knee OA, or no knee OA (Fig. 1) [32-34]. One-hundred and twenty-five knees had no radiographic OA (Kellgren-Lawrence [KL] grade $<2$ ) at the OAI baseline but then developed advanced-stage knee OA (KL grade 3 or 4 ) within 4 years. Knees from the other 2 groups were randomly matched by sex to the cases in a 1:1 ratio. Common knee OA was defined as no radiographic knee OA at baseline and progressed in $\mathrm{KL}$ grade $\geq 1$ but did not reach advanced-stage knee OA over the course of 4 years. 


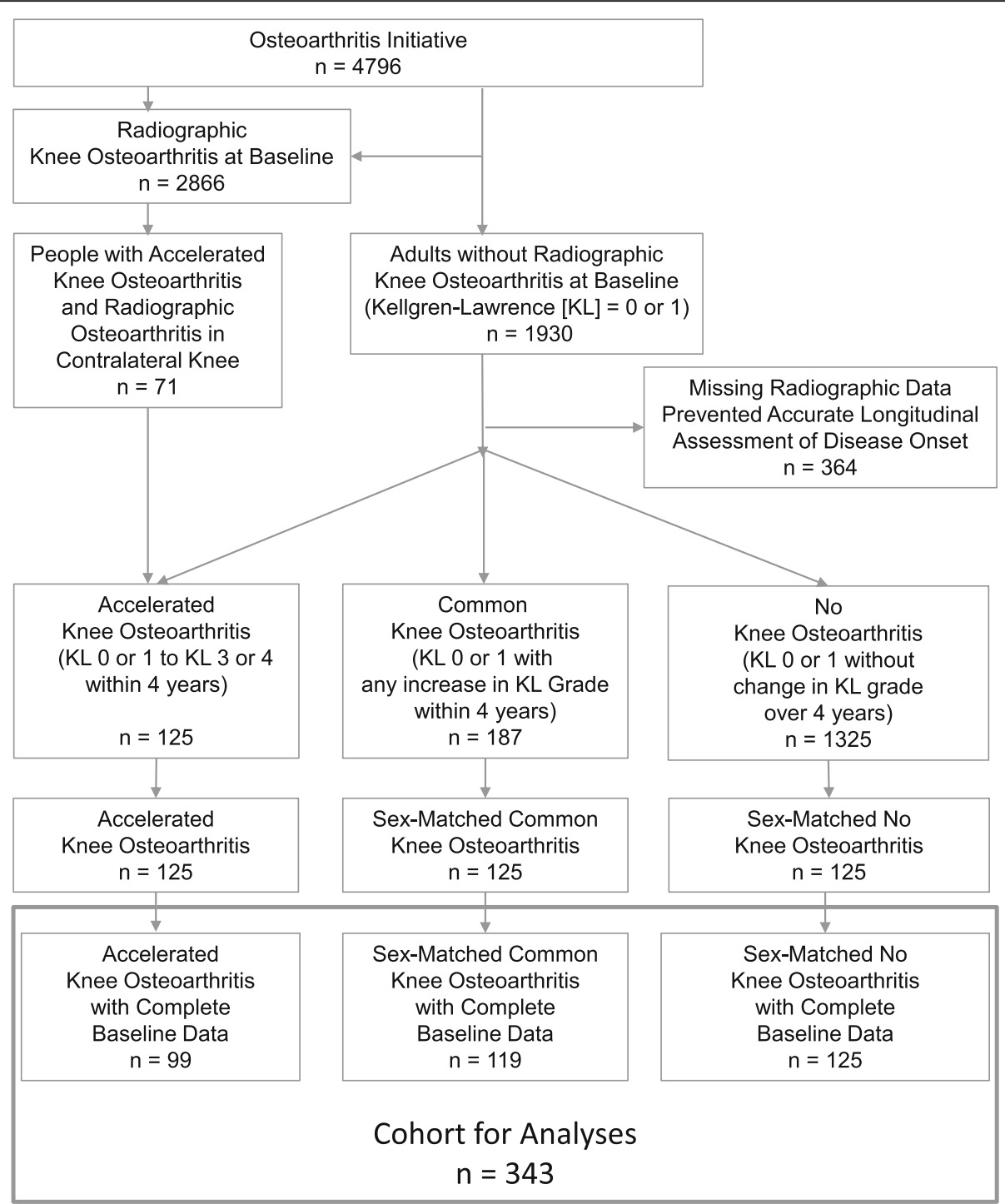

Fig. 1 Flow chart of eligibility for participants in analyses

No knee OA was defined as no radiographic knee OA at baseline and had no change in KL grade between the baseline visit and 4 years thereafter. From the 3 groups ( $n=375$ ), we included 343 individuals who had sagittal intermediate-weighted, turbo spin echo, fat-suppressed magnetic resonance (MR) images and fasting serum data available at baseline (accelerated knee OA: $n=99$, common knee OA: $n=119$, no knee OA: $n=125$ ). This sample of participants offered us the advantage of a rich dataset and enabled us to ensure an adequate sample size of knees had baseline BMLs and effusion.

We included one knee per person. Among those who progressed over the subsequent 4 years, we included the knee with the greatest radiographic progression. For individuals with no radiographic progression, we selected a knee to match one person of the same sex who developed advanced-stage radiographic knee OA.

\section{MR image acquisition}

To assess BMLs and knee effusion volume we used sagittal intermediate-weighted, turbo spin echo, fatsuppressed MR images which were acquired at the OAI baseline visit (field of view $=160 \mathrm{~mm}$, slice thickness = $3 \mathrm{~mm}$, skip $=0 \mathrm{~mm}$, flip angle $=180$ degrees, echo time $=30 \mathrm{~ms}$, recovery time $=3200 \mathrm{~ms}, 313 \times 448$ matrix (interpolated to $512 \times 512$ ), phase encode superior/inferior, $\times$ resolution $=0.357 \mathrm{~mm}$, and $\mathrm{y}$ resolution $=$ $0.511 \mathrm{~mm}$ ) [35]. All MR sequences were taken using one of four identical Siemens Trio 3-Tesla MR systems and a USA Instruments quadrature transmit-receive knee coil 
at one of the four OAI clinical sites. The OAI protocol for MR imaging is available on the OAI website [31].

\section{BML measurements}

A single reader (ACS) used a semi-automated program, which we described previously [36-38], to measure BMLs in the distal femur and proximal tibia on sagittal intermediate-weighted, turbo spin echo, fat-suppressed MR images. First, the reader identified the first and last slices in the MR sequence in which the tibia or femur are present. Following this, the reader marked several points along the articular surface of both the tibia and femur to define the crude boundaries of each bone. To mark the border furthest from the articular surface the reader marked the bone just prior to the epiphyseal line. Once the reader completed the manual steps, the program automatically segmented the bone boundary and then the areas of high-signal intensity within the bone by performing a thresholding and curve evolution process twice. These areas of high-signal intensity represented a probable BML. We then used two criteria to exclude false-positive regions and to define a BML: (1) the distance between a BML and the articular surface should be $\leq 10 \mathrm{~mm}$ [39-41] and (2) a BML should appear on $>1$ MR image. The program output the BML volume for 4 different regions: medial femur, lateral femur, medial tibia, and lateral tibia.

Our reader had good reliability (intra-tester ICC 3,1 model baseline $B M L=0.86$ to 0.97 ). The reader also had good reliability compared with previous readers in our team (inter-tester intraclass correlation coefficient; ICC 2,1 model: baseline $\mathrm{BML}=0.75$ to 0.93$)$. A second reader (JBD) reviewed all images for quality control, assuring that bone segmentation remained consistent across knees and time.

\section{Effusion measurements}

Two readers measured knee effusion volume on sagittal intermediate-weighted, turbo spin echo, fat-suppressed MR images using a semi-automated program. Since the MR images were acquired without contrast the volumetric measurement may have included knee effusion and synovitis; however, throughout this manuscript we will describe the outcome as knee effusion volume. The program required the reader to first mark the first and last slice of the MR sequence in which the tibia or femur are visible. The reader then marked the base of the patella and the distal attachment of the patellar ligament into the tibial tuberosity on a middle slice. Following this, the program automatically applied a threshold to segment the effusion volume (areas of high-signal intensity) within the entire knee. The user then reviewed each slice with the option to manually adjust the threshold or remove areas of high-signal intensity that were not effusion (e.g., subchondral bone cysts).

Two readers (JBD and FA) used the semi-automated software to quantify changes in MR-based knee effusion. Intra-tester reliability was good (ICC 3,1 model): reader 1 (JBD) baseline $=0.96$; reader2 [FA] baseline $=0.84$ ). Since inter-tester reliability was $=0.42$, reader 1 conducted a quality review of all segmented images to ensure consistent results between readers, knees, and time. The final data was based on the reviewed segmentation results that were corrected by reader 1 .

\section{Biospecimens collection}

Participation in the OAI was contingent upon a baseline fasting blood draw. Participants were asked to fast for $>8 \mathrm{~h}$ prior to their study visit. Trained staff performed baseline blood draws at least $1 \mathrm{~h}$ after the participant had gotten up in the morning.

Immediately after collection, study staff inverted the serum sample tubes and kept them at room temperature for $30 \mathrm{~min}$. Following these $30 \mathrm{~min}$, the tubes were centrifuged at $4{ }^{\circ} \mathrm{C}$ for a total of 30,000 g-minutes and then immediately transferred to cryovials. Within $15 \mathrm{~min}$ of being transferred to cryovials the samples were placed in $\mathrm{a}-70{ }^{\circ} \mathrm{C}$ freezer for at least $30 \mathrm{~min}$ prior to being shipped to Fisher Bioservices for long-term storage. In September 2015, Fisher Bioservices shipped the samples to Temple University School of Medicine. Additional details regarding the collection of biospecimens can be found in the OAI protocols, which are available on the OAI website [31].

\section{Biospecimens assay}

All assays were performed at Temple University School of Medicine within 2 months of delivery. The samples were de-identified to ensure the laboratory staff was blinded. To assess serum high-sensitivity CRP, an enzyme-linked immunosorbent assay was used (ELISA, Novex by Life Technology, Carlsbad, CA; range = $18.75-1200 \mathrm{pg} / \mathrm{mL}$, sensitivity $=18.75 \mathrm{pg} / \mathrm{mL}$, coefficient of variation $<7.52 \%$ for intra-assay precision, and coefficient of variation $<10 \%$ for inter-assay precision). To assay GSP, an ELISA kit was used (MyBioSource, San Diego, CA; range $=78.125 \mu \mathrm{M}-1000 \mathrm{nmol} / \mathrm{mL}$, sensitivity $=48.875 \mu \mathrm{M} / \mathrm{mL}$, and coefficient of variation $<10 \%)$. Samples used for glucose analyses were deproteinized prior to analysis with $10 \mathrm{kD}$ Spin Columns (Abcam, Cambridge, MA), per manufacturer's directions. For fasting glucose levels, Glucose Assay Kits were used (Abcam; range $=1-1000 \mu \mathrm{M}$, sensitivity $=1 \mu \mathrm{M} / \mathrm{mL}$, and coefficient of variation $<2 \%)$. All samples were tested in duplicate. 


\section{Other clinical variables}

We extracted potential confounders from the public OAI data files: age, sex, body mass index (BMI), and Physical Activity Scale for the Elderly (PASE) scores (Files: enrollees, version 22; allclinical00, version 0.2.2). We also extracted self-reported diabetes from the Charlson Comorbidity Index. All variables were collected based on standardized procedures, which are defined in the OAI protocols, which are available on the OAI website (15).

\section{Statistical analyses}

Initially, we calculated descriptive statistics; explored the distribution of CRP, GSP, and glucose concentrations, and applied the natural $\log (\mathrm{ln})$ transformation for GSP to stabilize the variable. Since our BML segmentation program detected small areas of increased signal intensity on every knee, we classified a knee as having a prevalent BML if any region (medial femur, medial tibia, lateral femur, lateral tibia) had a BML volume $>1 \mathrm{~cm}^{3}$. The BML cut-point was based on a prior classification and regression tree analysis with medial joint space narrowing progression as an outcome [37, 42]. A knee was classified as having prevalent effusion if the knee effusion volume was greater than the median knee effusion volume $\left(>7.5 \mathrm{~cm}^{3}\right)$.

To assess the relationship between serum measurements (predictors) and BMLs or knee effusion (outcomes) we used logistic regression models, adjusting for age, sex, BMI, and PASE score. In separate models, we explored interactions between the biomarkers and BMI. We checked for a linear relationship between the three predictors and the log odds for the presence of BMLs or effusion using a plot spline transformation with linear hypothesis test (SAS macro: \%PSPLINET, 3 knots). When a nonlinear relationship was present we performed piece-wise logistic regression analyses and selected the cut-point based on spline graphs.

We conducted 2 sensitivity analyses: 1 ) adjusting for self-reported smoking ever in life and 2) only including people who reported no diabetes. We also explored if there was an interaction between sex and any of the three serum measurements.
All analyses were performed with SAS Enterprise Guide 7.13 (Cary, NC).

\section{Results}

We had complete data for 343 participants. The cohort had a mean age of $59 \pm 9$ years, BMI of $27.9 \pm 4.4 \mathrm{~kg} / \mathrm{m}^{2}$, PASE score of $171 \pm 82$, and $64 \%$ were female. Only 10 participants reported diabetes on the Charlson Comorbidity Index (7 had missing data). Overall, the sample had a mean (standard deviation) total BML volume $0.92(1.48) \mathrm{cm}^{3}$, effusion volume $9.09(6.28) \mathrm{cm}^{3}$, CRP 3.31 (1.21) mg/L, lnGSP 5.48 (0.72), and glucose 110.19 (26.52) $\mathrm{mg} / \mathrm{dL}$.

Only CRP concentrations were associated with the presence of BMLs $(\mathrm{OR}=1.43,95 \% \mathrm{CI}=1.09$ to 1.87 ; Table 1). There were no associations between GSP or glucose levels and the presence of BMLs.

We found a statistical interaction between BMI and CRP when assessing effusion: among adults with a BMI $<25 \mathrm{~kg} / \mathrm{m}^{2}$, there was a trend for a positive association between CRP and knee effusion (OR $=1.40,95 \%$ CI 1.00 to 1.97) but this association was null for overweight or obese individuals $(\mathrm{OR}=0.83,95 \% \mathrm{CI}=0.65$ to 1.07$)$. We detected a nonlinear relationship between lnGSP levels and the presence of baseline effusion: for lnGSP levels $\geq 5.5 \mathrm{~cm}^{3}$, individuals with greater GSP concentrations were more likely to have baseline effusion $(O R=2.02)$. In contrast, for $\operatorname{lnGSP}$ levels $<5.5 \mathrm{~cm}^{3}$, individuals with lower GSP concentrations were more likely to have effusion $(\mathrm{OR}=0.39$; see Table 2). Fasting glucose levels were not significantly associated with the presence of baseline effusion.

In sensitivity analyses, we found that adjusting for smoking or limiting the sample to people with no diabetes had little influence on the estimated odds ratios $(<10 \%$ change in OR). We found no significant interactions with sex.

\section{Discussion}

Among adults without knee OA, we found that people with greater systemic inflammation (CRP concentrations) were more likely to have a BML. Furthermore, among individuals with a normal weight, adults with high CRP

Table 1 C-reactive protein is associated with the prevalence of bone marrow lesions (BMLs)

\begin{tabular}{|c|c|c|c|c|}
\hline Predictor (continuous) & $\begin{array}{l}\text { No BML } \\
(n=287) \\
\text { Mean (SD) } \\
\text { (Reference) }\end{array}$ & $\begin{array}{l}\text { Prevalent BML } \\
(n=56) \\
\text { Mean (SD) }\end{array}$ & $\begin{array}{l}\text { Prevalent BML } \\
\text { Crude OR }(95 \% \mathrm{Cl}), p \text {-value } \\
\text { (per unit of biomarker concentration) }\end{array}$ & $\begin{array}{l}\text { Prevalent BML } \\
\text { Adjusted OR }(95 \% \mathrm{Cl}), \text { p-value } \\
\text { (per unit of biomarker concentration) }\end{array}$ \\
\hline C-reactive Protein (mg/L) & $3.24(1.22)$ & $3.67(1.12)$ & $1.36(1.05,1.75), 0.02$ & $1.43(1.09,1.87), 0.01$ \\
\hline Glycated serum protein (log) & $5.50(0.70)$ & $5.43(0.82)$ & $0.87(0.58,1.32), 0.52$ & $0.97(0.65,1.46), 0.90$ \\
\hline Glucose $(\mathrm{mg} / \mathrm{dL})$ per $10 \mathrm{mg} / \mathrm{dL}$ & $109.3(27.2)$ & $114.6(22.2)$ & $1.08(0.97,1.20), 0.18$ & $1.07(0.95,1.19), 0.28$ \\
\hline
\end{tabular}

Adjusted analyses include age, body mass index, sex, and Physical Activity Scale for the Elderly Score. OR odds ratio, $95 \%$ CI $95 \%$ confidence interval. Bold represents statistically significant findings $(p<0.05)$ 
Table 2 Glycated serum protein is associated with the prevalence of effusion

\begin{tabular}{|c|c|c|c|c|}
\hline Predictor (continuous) & $\begin{array}{l}\text { No-Little Effusion } \\
(n=173) \\
\text { Mean (SD) } \\
\text { (Reference) }\end{array}$ & $\begin{array}{l}\text { Effusion }\left(\geq 7.5 \mathrm{~cm}^{3}\right) \\
(n=170) \\
\text { Mean (SD) }\end{array}$ & $\begin{array}{l}\text { Prevalent Effusion } \\
\text { Crude OR }(95 \% \mathrm{Cl}), \text { } \text {-value } \\
\text { (per unit of biomarker concentration) }\end{array}$ & $\begin{array}{l}\text { Prevalent Effusion } \\
\text { Adjusted OR }(95 \% \mathrm{Cl}), p \text {-value } \\
\text { (per unit of biomarker concentration) }\end{array}$ \\
\hline $\mathrm{CRP}(\mathrm{mg} / \mathrm{L})$ & $3.29(1.27)$ & $3.34(1.16)$ & $1.04(0.87,1.24), 0.68$ & $1.01(0.83,1.22), 0.93$ \\
\hline $\operatorname{lnGSP}<5.5$ & $5.10(0.29)$ & $4.99(0.40)$ & $0.36(0.18,0.73), 0.004$ & $0.39(0.18,0.83), 0.01$ \\
\hline $\ln G S P \geq 5.5$ & $6.01(0.49)$ & $6.29(0.71)$ & $2.01(1.22,3.32), 0.01$ & $2.02(1.22,3.34), 0.01$ \\
\hline Glucose (mg/dL) per $10 \mathrm{mg} / \mathrm{dL}$ & $108.7(27.9)$ & $111.7(25.1)$ & $1.04(0.96,1.13), 0.31$ & $1.03(0.95,1.12), 0.48$ \\
\hline
\end{tabular}

All analyses were adjusted for age, body mass index, sex, and Physical Activity Scale for the Elderly score. CRP C-reactive Protein, InGSP glycated serum protein (log), OR odds ratio, $95 \% \mathrm{Cl} 95 \%$ confidence interval. Bold represents statistically significant findings $(p<0.05)$

concentrations may be more likely to have knee effusion. Finally, GSP concentrations had a "U-shaped" relationship with effusion. These findings highlight the complex relationships that systemic inflammation and impaired glucose homeostasis may have among knees without OA.

Our results complement a prior study of people with knee OA in which the authors reported positive associations between quartiles of CRP and the prevalence and worsening of knee BMLs over 2 years [28]. We went beyond quartile readings and looked at CRP as a continuous variable, finding a linear relationship between CRP concentrations and BML presence. While Zhu, et al. [28] collected data from participants with symptomatic knee OA, our study focused on individuals without knee $\mathrm{OA}$, but with established risk factors for the disease.

Only one study has looked at the relationship between glucose and BMLs. Specifically, the authors found a positive association between glucose levels and incident BMLs in women but not in men [30]; however, fasting glucose samples were collected $>9$ years prior to the initial MR images that were used for BML measurements. In contrast, we examined glucose concentrations in serum collected during the same visit as the MR images. Additionally, a novel aspect of our study was that we looked at a more stable metric of glucose homeostasis, GSP, rather than focusing purely on fasting glucose levels.

The inclusion of GSP in our analyses proved insightful as we found associations between GSP levels and the presence of baseline effusion. Our results complement our previous study that found a U-shaped correlation between GSP concentrations and incident knee OA: specifically, individuals with $\operatorname{lnGSP}$ concentrations $<5.7$ and individuals with $\operatorname{lnGSP}$ concentrations $>5.7$ were more likely to develop incident knee OA than those with lnGSP concentrations closer to 5.7 [32]. This U-shaped relationship may indicate that there are two clinically relevant subsets with effusion that warrant more study those with low GSP concentrations and others with high GSP concentrations. Increased formation of advanced glycation end-products leads to increased collagen cross-linking, which can make the cartilage more brittle.
This in turn can induce cartilage fragmentation [43], which could aggravate the synovium causing an increase in effusion volume [44, 45]. This may help explain the positive association between high GSP levels and effusion. Alternatively, the presence of knee effusion may lead to joint symptoms, which leads to a more sedentary life style and consequently a greater risk for impaired glucose homeostasis. Future studies should explore the cause for this association, as well as why low GSP levels are associated with effusion.

We also observed a trend that CRP had a positive association with effusion among individuals with a normal weight $\left(B M I<25 \mathrm{~kg} / \mathrm{m}^{2}\right.$ ) but not among adults who were overweight or obese. This may indicate that other factors besides systemic inflammation contribute to effusion among individuals who are overweight or obese. The increased joint load in an overweight or obese individual can cause cartilage damage [46], and cartilage fragments may aggravate the synovium $[41,45]$. It is important to note that the cross-sectional nature precludes us from determining if the presence of effusion - or other pathologies that contribute to effusion - may contribute to greater systemic inflammation among normal weight adults but not among individuals who are overweight and obese.

There were several potential limitations to our study. With our limited sample size, we may not have adequate power to explore more interactions, especially with the presence of nonlinear relationships. Despite this limitation, we detected nonlinear patterns and interactions that may be important to future research. It is vital that future studies anticipate non-linear relationships between glucose homeostasis and effusion. Secondly, our study did not consider the association between local inflammatory biomarkers and BMLs or effusion. We chose to focus solely on the whole-body burden of glucose and inflammatory homeostasis. There are many systemic and local factors that may contribute to changes in glucose and inflammatory homeostasis. Hence, we cannot infer causation; however, despite other possible causes for altered glucose and inflammatory homeostasis, BMLs, and effusion they are associated. Thirdly, the cross-sectional 
nature precludes us from making any causal inferences or disentangling whether systemic inflammation or glucose homeostasis leads the joint to be susceptible to BMLs or effusion or if the presence of BMLs and effusion may contribute to changes in systemic inflammation or glucose homeostasis. Finally, we used a sample that was enriched to include people who later develop radiographic knee $\mathrm{OA}$ and not a population-based sample, which would enable results that are more generalizable. Future population-based studies may build on these findings. Despite these limitations, we found novel associations and interactions that warrant further study.

\section{Conclusions}

We found that systemic inflammation, specifically CRP, may be associated with BMLs and effusion in normal weight individuals. Furthermore, glucose homeostasis, determined using GSP, may be associated with effusion. Though our results indicate that inflammatory and glucose homeostasis are related to structural features of $\mathrm{OA}$, it will be valuable to determine whether inflammatory and glucose homeostasis are related to incident symptomatic OA. Further understanding of these associations may help us develop strategies to prevent or delay symptomatic OA or alternatively to prevent an adverse influence of knee OA on systemic inflammation and impaired glucose homeostasis.

\section{Abbreviations}

BMI: Body mass index; BML: Bone marrow lesion; Cl: Confidence interval; CRP: C-reactive protein; GSP: Glycated serum protein; ICC: Intraclass correlation coefficient; MR: Magnetic resonance; OA: Osteoarthritis; OAl: Osteoarthritis Initiative; OR: Odds ratio; PASE: Physical Activity Scale for the Elderly

\section{Acknowledgements}

Not applicable.

\section{Funding}

These analyses were financially supported by the National Institute of Arthritis and Musculoskeletal and Skin Diseases of the National Institutes of Health under Award Number R01 AR065977. The OAI is a public-private partnership comprised of five contracts (N01-AR-2-2258; N01-AR-2-2259; N01-AR-2-2260; N01-AR-2-2261; N01-AR-2-2262) funded by the National Institutes of Health, a branch of the Department of Health and Human Services, and conducted by the OAI Study Investigators. Private funding partners include Merck Research Laboratories; Novartis Pharmaceuticals Corporation, GlaxoSmithKline; and Pfizer, Inc. Private sector funding for the OAl is managed by the Foundation for the National Institutes of Health. This manuscript was prepared using an OAl public use data set and does not necessarily reflect the opinions or views of the OAI investigators, the $\mathrm{NIH}$, or the private funding partners. This work was also supported in part by the Houston Veterans Affairs Health Services Research and Development Center of Excellence (HFP90-020). The views expressed in this article are those of the authors and do not necessarily represent the views of the Department of Veterans Affairs.

\section{Availability of data and materials}

The datasets generated and analyzed during the current study are available on the OAI website (https://oai.epi-ucsf.org/). Biochemical and MR-based measurements will be released on the OAI website but will also be available from the corresponding author on reasonable request.

\section{Authors' contributions}

ACS contributed to the acquisition of data, analysis and interpretation of data, drafting/revisions of article, as well as final approval of the article. MFB contributed to the conception and design, acquisition of data, interpretation of data, drafting/revisions of article, as well as final approval of the article. CBE contributed to the conception and design, acquisition of data, analysis and interpretation of data, drafting/revisions of article, as well as final approval of the article. MA contributed to the acquisition of data, interpretation of data, drafting/revisions of article, as well as final approval of the article. FA contributed to the acquisition of data, drafting/revisions of article, as well as final approval of the article. LLP contributed to the analysis and interpretation of data, drafting/revisions of article, as well as final approval of the article. BL contributed to the conception and design, analysis and interpretation of data, drafting/revisions of article, as well as final approval of the article. GHL contributed to the analysis and interpretation of data, drafting/revisions of article, as well as final approval of the article. MZ contributed to acquisition of data, interpretation of data, drafting/revisions of article, as well as final approval of the article. JP contributed to acquisition of data, interpretation of data, drafting/revisions of article, as well as final approval of the article. TEM contributed to the conception and design, acquisition of data, analysis and interpretation of data, drafting/revisions of article, as well as final approval of the article. JBD contributed to the conception and design, acquisition of data, analysis and interpretation of data, drafting/revisions of article, as well as final approval of the article.

\section{Competing interest}

JBD and MFB are members of the Editorial Board of BMC Musculoskeletal Disorders. The other authors declare that they have no competing interests.

\section{Ethics approval and consent to participate}

The OAl study was approved by institutional review boards at each OAI clinical site and the coordinating center: Memorial Hospital of Rhode Island Institutional Review Board, The Ohio State University's Biomedical Sciences Institutional Review Board, University of Pittsburgh Institutional Review Board, University of Maryland Baltimore - Institutional Review Board, and Committee on Human Research at University of California, San Francisco. All participants provided informed consent.

\section{Consent for publication}

Not applicable.

\section{Publisher's Note}

Springer Nature remains neutral with regard to jurisdictional claims in published maps and institutional affiliations.

\section{Author details \\ ${ }^{1}$ Division of Rheumatology, Tufts Medical Center, 800 Washington Street, Box \#406, Boston, MA 02111, USA. ²Department of Anatomy and Cell Biology, Temple University School of Medicine, 3500 North Broad Street, Philadelphia, PA 19140, USA. ${ }^{3}$ Center for Primary Care and Prevention, Alpert Medical School of Brown University, 111 Brewster Street, Pawtucket, RI 02860, USA. ${ }^{4}$ The Institute for Clinical Research and Health Policy Studies, Tufts Medical Center, 800 Washington Street, Box \#63, Boston, MA 02111, USA. ${ }^{5}$ Tufts Clinical and Translational Science Institute, Tufts University, 800 Washington Street, Box \#63, Boston, MA 02111, USA. ${ }^{6}$ Brigham \& Women's Hospital and Harvard Medical School, 75 Francis Street PBB-B3, Boston, MA 02115, USA. ${ }^{7}$ Medical Care Line and Research Care Line, Houston Health Services Research and Development (HSR\&D) Center of Excellence Michael E. DeBakey VAMC, 2002 Holcombe Blvd, Houston, TX 77030, USA. ${ }^{8}$ Section of Immunology, Allergy, and Rheumatology, Baylor College of Medicine, 1 Baylor Plaza, BCM-285, Houston, TX 77030, USA. ${ }^{9}$ Department of Electrical Engineering, Tufts University, 161 College Avenue, Medford, MA 02155, USA.}

Received: 19 September 2017 Accepted: 22 December 2017

Published online: 05 January 2018

\section{References}

1. Richmond SA, Fukuchi RK, Ezzat A, Schneider K, Schneider G, Emery CA. Are joint injury, sport activity, physical activity, obesity, or occupational activities predictors for osteoarthritis? A systematic review. J Orthop Sports Phys Ther. 2013;43(8):515-b519. 
2. Oliveria SA, Felson DT, Reed JI, Cirillo PA, Walker AM. Incidence of symptomatic hand, hip, and knee osteoarthritis among patients in a health maintenance organization. Arthritis Rheum. 1995;38(8):1134-41.

3. Jiang L, Tian W, Wang Y, Rong J, Bao C, Liu Y, Zhao Y, Wang C. Body mass index and susceptibility to knee osteoarthritis: a systematic review and meta-analysis. Joint Bone Spine. 2012;79(3):291-7.

4. Blagojevic M, Jinks C, Jeffery A, Jordan KP. Risk factors for onset of osteoarthritis of the knee in older adults: a systematic review and metaanalysis. Osteoarthr Cartil. 2010;18(1):24-33.

5. Rosa SC, Rufino AT, Judas FM, Tenreiro CM, Lopes MC, Mendes AF. Role of glucose as a modulator of anabolic and catabolic gene expression in normal and osteoarthritic human chondrocytes. J Cell Biochem. 2011; 112(10):2813-24.

6. Chen HC, Shah S, Stabler TV, Li YJ, Kraus VB. Biomarkers associated with clinical phenotypes of hand osteoarthritis in a large multigenerational family: the CARRIAGE family study. Osteoarthr Cartil. 2008;16(9):1054-9.

7. Elahi D, Muller DC, Tzankoff SP, Andres R, Tobin JD. Effect of age and obesity on fasting levels of glucose, insulin, glucagon, and growth hormone in man. J Gerontol. 1982;37(4):385-91.

8. Lucey AJ, Paschos GK, Thorsdottir I, Martinez JA, Cashman KD, Kiely M. Young overweight and obese women with lower circulating osteocalcin concentrations exhibit higher insulin resistance and concentrations of Creactive protein. Nutrition Res (New York, NY). 2013;33(1):67-75.

9. Lee SW, Jo HH, Kim MR, You YO, Kim JH. Association between obesity, metabolic risks and serum osteocalcin level in postmenopausal women. Gynecol Endocrinol. 2012;28(6):472-7.

10. Driban JB, Barr AE, Amin M, Sitler MR, Barbe MF. Joint inflammation and early degeneration induced by high force reaching are attenuated by ibuprofen in an animal model of work-related musculoskeletal disorder. J Biomed Biotechnol. 2011;2011:691412.

11. Kerkhof HJ, Bierma-Zeinstra SM, Castano-Betancourt MC, de Maat MP, Hofman A, Pols HA, Rivadeneira F, Witteman JC, Uitterlinden AG, van Meurs JB. Serum $C$ reactive protein levels and genetic variation in the CRP gene are not associated with the prevalence, incidence or progression of osteoarthritis independent of body mass index. Ann Rheum Dis. 2010; 69(11):1976-82.

12. Labouesse MA, Gertz ER, Piccolo BD, Souza EC, Schuster GU, Witbracht MG, Woodhouse LR, Adams SH, Keim NL, Van Loan MD. Associations among endocrine, inflammatory, and bone markers, body composition and weight loss induced bone loss. Bone. 2014;64:138-46.

13. Jonsson H, Helgadottir GP, Aspelund T, Eiriksdottir G, Sigurdsson S, Siggeirsdotti K, Ingvarsson T, Harris TB, Launer L, Gudnason V. Hand osteoarthritis severity is associated with Total knee joint replacements independently of BMI. The agesReykjavik study. Open Rheumatol J. 2011;5:7-12.

14. Kraus VB, Stabler TV, Luta G, Renner JB, Dragomir AD, Jordan JM. Interpretation of serum C-reactive protein (CRP) levels for cardiovascular disease risk is complicated by race, pulmonary disease, body mass index, gender, and osteoarthritis. Osteoarthr Cartil. 2007;15(8):966-71.

15. Fraenkel $L$, Roubenoff R, LaValley M, McAlindon T, Chaisson C, Evans S, Harris T, Dinarello CA, Felson DT. The association of peripheral monocyte derived interleukin 1 beta (IL-1 beta), IL-1 receptor antagonist, and tumor necrosis factor-alpha with osteoarthritis in the elderly. J Rheumatol. 1998; 25(9):1820-6.

16. Hart DJ, Doyle DV, Spector TD. Association between metabolic factors and knee osteoarthritis in women: the Chingford study. J Rheumatol. 1995;22(6): 1118-23.

17. Xu L, Hayashi D, Roemer FW, Felson DT, Guermazi A. Magnetic resonance imaging of subchondral bone marrow lesions in association with osteoarthritis. Semin Arthritis Rheum. 2012;42(2):105-18.

18. Yusuf E, Kortekaas MC, Watt I, Huizinga TW, Kloppenburg M. Do knee abnormalities visualised on MRI explain knee pain in knee osteoarthritis? A systematic review. Ann Rheum Dis. 2011;70(1):60-7.

19. Roemer FW, Kwoh CK, Hannon MJ, Hunter DJ, Eckstein F, Fujii T, Boudreau RM, Guermazi A. What comes first? Multitissue involvement leading to radiographic osteoarthritis: magnetic resonance imaging-based trajectory analysis over four years in the osteoarthritis initiative. Arthritis Rheumatology. 2015;67(8):2085-96.

20. Sharma L, Chmiel JS, Almagor O, Dunlop D, Guermazi A, Bathon JM, Eaton CB, Hochberg MC, Jackson RD, Kwoh CK, et al. Significance of preradiographic magnetic resonance imaging lesions in persons at increased risk of knee osteoarthritis. Arthritis Rheumatol. 2014;66(7):1811-9.
21. Felson DT, Parkes MJ, Marjanovic EJ, Callaghan M, Gait A, Cootes T, Lunt M, Oldham J, Hutchinson CE. Bone marrow lesions in knee osteoarthritis change in 6-12 weeks. Osteoarthr Cartil. 2012;20(12):1514-8.

22. Brandt KD, Mazzuca SA, Buckwalter KA. Acetaminophen, like conventional NSAIDs, may reduce synovitis in osteoarthritic knees. Rheumatology (Oxford, England). 2006:45(11):1389-94.

23. Laslett LL, Dore DA, Quinn SJ, Boon P, Ryan E, Winzenberg TM, Jones G. Zoledronic acid reduces knee pain and bone marrow lesions over 1 year: a randomised controlled trial. Ann Rheum Dis. 2012;71(8):1322-8.

24. Callaghan MJ, Parkes MJ, Hutchinson CE, Gait AD, Forsythe LM, Marjanovic EJ, Lunt M, Felson DT. A randomised trial of a brace for patellofemoral osteoarthritis targeting knee pain and bone marrow lesions. Ann Rheum Dis. 2015;74(6):1164-70.

25. Visser M, Bouter LM, McQuillan GM, Wener MH, Harris TB. Elevated Creactive protein levels in overweight and obese adults. JAMA. 1999;282(22): 2131-5

26. Jin X, Beguerie JR, Zhang W, Blizzard L, Otahal P, Jones G, Ding C. Circulating $C$ reactive protein in osteoarthritis: a systematic review and meta-analysis. Ann Rheum Dis. 2015;74(4):703-10.

27. Saberi Hosnijeh F, Siebuhr AS, Uitterlinden AG, Oei EHG, Hofman A, Karsdal MA, Bierma-Zeinstra SM, Bay-Jensen AC, van Meurs JBJ. Association between biomarkers of tissue inflammation and progression of osteoarthritis: evidence from the Rotterdam study cohort. Arthritis Res Ther. 2016;18(1):1-10.

28. Zhu Z, Jin X, Wang B, Wluka A, Antony B, Laslett LL, Winzenberg T, Cicuttini $F$, Jones $G$, Ding $C$. Cross-sectional and longitudinal associations between serum levels of hs-CRP, knee bone marrow lesions and knee pain in patients with knee osteoarthritis. Arthritis Care Res. 2016;68(10):1471-7.

29. Stannus OP, Jones G, Blizzard L, Cicuttini FM, Ding C. Associations between serum levels of inflammatory markers and change in knee pain over 5 years in older adults: a prospective cohort study. Ann Rheum Dis. 2013;72(4):535-40.

30. Davies-Tuck ML, Wang Y, Wluka AE, Berry PA, Giles GG, English DR, Cicuttini FM. Increased fasting serum glucose concentration is associated with adverse knee structural changes in adults with no knee symptoms and diabetes. Maturitas. 2012;72(4):373-8.

31. The Osteoarthritis Initiative. http://oai.epi-ucsf.org/. Accessed 5 Dec 2017.

32. Driban JB, Eaton CB, Amin M, Stout AC, Price LL, Lu B, Lo GH, McAlindon TE, Barbe MF. Glucose homeostasis influences the risk of incident knee osteoarthritis: data from the osteoarthritis initiative. J Orthop Res. 2017; 35(10):2282-7. doi:https://doi.org/10.1002/jor.23531.

33. Driban JB, Stout AC, Duryea J, Lo GH, Harvey WF, Price LL, Ward RJ, Eaton CB, Barbe MF, Lu B, et al. Coronal tibial slope is associated with accelerated knee osteoarthritis: data from the osteoarthritis initiative. BMC Musculoskelet Disord. 2016;17:299.

34. Driban JB, McAlindon TE, Amin M, Price LL, Eaton CB, Davis JE, Lu B, Lo GH, Duryea J, Barbe MF. Risk factors can classify individuals who develop accelerated knee osteoarthritis: data from the osteoarthritis initiative. $J$ Orthop Res. 2017. doi:https://doi.org/10.1002/jor.23675. [Epub ahead of print].

35. Peterfy CG, Schneider E, Nevitt M. The osteoarthritis initiative: report on the design rationale for the magnetic resonance imaging protocol for the knee. Osteoarthr Cartil. 2008;16(12):1433-41.

36. Pang J, Driban JB, Destenaves G, Miller E, Lo GH, Ward RJ, Price LL, Lynch $J A$, Eaton CB, McAlindon TE. Quantification of bone marrow lesion volume and volume change using semi-automated segmentation: data from the osteoarthritis initiative. BMC Musculoskelet Disord. 2013;14(1):3.

37. Driban JB, Price L, Lo GH, Pang J, Hunter DJ, Miller E, Ward RJ, Eaton CB, Lynch JA, McAlindon TE. Evaluation of bone marrow lesion volume as a knee osteoarthritis biomarker-longitudinal relationships with pain and structural changes: data from the osteoarthritis initiative. Arthritis Res Ther 2013;15(5):R112.

38. Driban JB, Lo GH, Price L, Pang J, Miller E, Ward RJ, Hunter DJ, Eaton CB, Lynch JA, McAlindon TE. Bone marrow lesion volume reduction is not associated with improvement of other periarticular bone measures: data from the osteoarthritis initiative. Arthritis Res Ther. 2013;15(5):R153.

39. Driban JB, Lo GH, Lee JY, Ward RJ, Miller E, Pang J, Price LL, McAlindon TE. Quantitative bone marrow lesion size in osteoarthritic knees correlates with cartilage damage and predicts longitudinal cartilage loss. BMC Musculoskelet Disord. 2011;12:217.

40. Roemer FW, Frobell R, Hunter DJ, Crema MD, Fischer W, Bohndorf K, Guermazi A. MRI-detected subchondral bone marrow signal alterations of 
the knee joint: terminology, imaging appearance, relevance and radiological differential diagnosis. Osteoarthr Cartil. 2009;17(9):1115-31.

41. Roemer FW, Khrad H, Hayashi D, Jara H, Ozonoff A, Fotinos-Hoyer AK, Guermazi A. Volumetric and semiquantitative assessment of MRI-detected subchondral bone marrow lesions in knee osteoarthritis: a comparison of contrast-enhanced and non-enhanced imaging. Osteoarthr Cartil. 2010;18(8): 1062-6.

42. Antony B, Driban JB, Price LL, Lo GH, Ward RJ, Nevitt M, Lynch J, Eaton CB, Ding C, McAlindon TE. The relationship between meniscal pathology and osteoarthritis depends on the type of meniscal damage visible on magnetic resonance images: data from the osteoarthritis initiative. Osteoarthr Cartil. 2017;25(1):76-84

43. Li Y, Wei X, Zhou J, Wei L. The age-related changes in cartilage and osteoarthritis. Biomed Res Int. 2013;2013:916530.

44. Cucchiarini M, de Girolamo L, Filardo G, Oliveira JM, Orth P, Pape D, Reboul P. Basic science of osteoarthritis. J Exp Orthop. 2016;3(1):22.

45. Roemer FW, Guermazi A, Felson DT, Niu J, Nevitt MC, Crema MD, Lynch JA, Lewis CE, Torner J, Zhang Y. Presence of MRI-detected joint effusion and synovitis increases the risk of cartilage loss in knees without osteoarthritis at 30-month follow-up: the MOST study. Ann Rheum Dis. 2011;70(10):1804-9.

46. Berry PA, Wluka AE, Davies-Tuck ML, Wang Y, Strauss BJ, Dixon JB, Proietto J, Jones $\mathrm{G}$, Cicuttini FM. The relationship between body composition and structural changes at the knee. Rheumatology (Oxford, England). 2010; 49(12):2362-9.

\section{Submit your next manuscript to BioMed Central} and we will help you at every step:

- We accept pre-submission inquiries

- Our selector tool helps you to find the most relevant journal

- We provide round the clock customer support

- Convenient online submission

- Thorough peer review

- Inclusion in PubMed and all major indexing services

- Maximum visibility for your research

Submit your manuscript at www.biomedcentral.com/submit

) Biomed Central 